\title{
Cosmologically Safe QCD Axion without Fine-Tuning
}

\author{
Masaki Yamada, ${ }^{1,2,3}$ Tsutomu T. Yanagida, ${ }^{1}$ and Kazuya Yonekura ${ }^{1}$ \\ ${ }^{1}$ Kavli IPMU (WPI), UTIAS, The University of Tokyo, Kashiwa, Chiba 277-8583, Japan \\ ${ }^{2}$ Institute for Cosmic Ray Research, The University of Tokyo, Kashiwa, Chiba 277-8582, Japan \\ ${ }^{3}$ Deutsches Elektronen-Synchrotron DESY, 22607 Hamburg, Germany
}

(Received 6 November 2015; published 5 February 2016)

\begin{abstract}
Although QCD axion models are widely studied as solutions to the strong $C P$ problem, they generically confront severe fine-tuning problems to guarantee the anomalous Peccei-Quinn (PQ) symmetry. In this Letter, we propose a simple QCD axion model without any fine-tunings. We introduce an extra dimension and a pair of extra quarks living on two branes separately, which is also charged under a bulk Abelian gauge symmetry. We assume a monopole condensation on our brane at an intermediate scale, which implies that the extra quarks develop chiral symmetry breaking and the PQ symmetry is broken. In contrast to Kim's original model, our model explains the origin of the PQ symmetry thanks to the extra dimension and avoids the cosmological domain wall problem because of chiral symmetry breaking in Abelian gauge theory.
\end{abstract}

DOI: 10.1103/PhysRevLett.116.051801

Introduction.-The null result of a neutron electric dipole moment puts an upper bound on the magnitude of the strong $C P$ phase: $\theta_{\mathrm{QCD}} \lesssim 10^{-(10-11)}$ [1]. Such a small fundamental parameter is an outstanding mystery in particle physics, known as the strong $C P$ problem. Peccei and Quinn have proposed a mechanism to explain its smallness by introducing an anomalous global Abelian symmetry, called PQ symmetry [2,3]. PQ symmetry is assumed to be broken spontaneously at an intermediate scale, which predicts a pseudo-Nambu-Goldstone (NG) boson, called an axion [4]. The axion obtains a periodic potential due to the nonperturbative effect below the QCD scale, and its vacuum expectation value (VEV) cancels the unwanted $C P$ phase $[5,6]$. Here, in order to suppress the effective $C P$ phase sufficiently, the PQ symmetry breaking effects should be suppressed precisely. In general, however, quantum gravity effects may induce PQ symmetry breaking terms, so that severe fine-tunings may be required to explain the smallness of the strong $C P$ phase [7-13].

In Refs. [14,15], it has been revealed that a model with a flat extra dimension can explain the origin of PQ symmetry without any fine-tunings. (See Refs. [16-19] for other mechanisms to explain the origin of PQ symmetry.) The five-dimensional (5D) manifold is $R^{4} \times S^{1} / Z_{2}$, and two branes are located at the fixed points in the $S^{1} / Z_{2}$ orbifold. Each extra quark-antiquark pair lives on branes separately, which results in suppression of PQ symmetry breaking operators. Introducing a bulk strong gauge interaction under which the extra quarks are charged, one can consider the dynamical PQ symmetry breaking scenario [20]. A composite NG boson is identified with an axion and the strong $C P$ problem is solved without fine-tunings. Unfortunately, however, there are cosmological difficulties in this scenario. If the PQ symmetry is dynamically broken before primordial inflation and is never restored after inflation, the axion acquires quantum fluctuations during inflation and predicts axion isocurvature perturbation, which is severely constrained by the observations of cosmic microwave background (CMB) temperature fluctuations [21-23]. (In Ref. [24] a scenario has been proposed to solve the axion isocurvature problem in case PQ symmetry is broken before inflation.) On the other hand, if PQ symmetry is restored and then dynamically broken after inflation, e.g., in a high reheating temperature scenario, domain walls form at the QCD phase transition $[25,26]$. If the bulk strong interaction is $S U(N)_{H}$ gauge interaction, the domain wall number is $N$. In this case, domain walls are stable and soon dominate the energy density of the Universe after the QCD phase transition. Since the resulting Universe is highly inhomogeneous, this scenario is excluded. This is known as the axion domain wall problem.

Here, let us assume that chiral symmetry breaking is induced by a bulk $U(1)_{H}$ gauge interaction rather than $S U(N)_{H}$. In this case, the PQ symmetry is dynamically broken completely at the QCD phase transition, so that the domain wall number is unity. Since these domain walls are unstable due to their tension, there is no domain wall problem in this case. Therefore, we can safely consider the scenario that PQ symmetry is broken after inflation.

The next question is how chiral symmetry breaking occurs in Abelian gauge theory. Actually, there are many works related to the confinement and chiral symmetry breaking in Abelian-like gauge theories to reveal QCD confinement and chiral symmetry breaking. 't Hooft conjectured that long-distance physics could be realized only by Abelian degrees of freedom in QCD, which is called Abelian dominance [27]. The lattice QCD studies in fact reveal that the confinement and chiral symmetry breaking may occur with an Abelian-projected field in the maximally Abelian gauge [28-30], where a monopole current plays an 
important role [31,32]. These studies may imply that electron confining and chiral symmetry breaking can occur in Abelian gauge theory. 't Hooft's conjecture stands on an Abelian theory with a magnetic superconductor for confinement, where a physical string arises between an electron and an antielectron because of a monopole condensation [33]. Motivated by these studies, in this Letter we introduce a monopole on our brane and assume chiral symmetry breaking of electrons (extra quarks) by the condensation of the monopole.

Our model with a monopole and electrons may also be supported by Refs. [34-37], where the authors have discovered UV complete theories with monopoles and electrons. They consider an $S U(2)$ gauge theory with at most three flavor electrons in $N=2$ supersymmetry. In an $N=2$ supersymmetric multiplet, $S U(2)$ gluons are accompanied by Dirac fermions and complex scalars in the adjoint representation of the gauge group. The moduli space of this theory has a branch called the Coulomb branch, on which the complex scalar component of the gauge multiplet has a nonzero VEV and the $S U(2)$ gauge symmetry is spontaneously broken down to $U(1)$. As a result, a monopole appears in the low energy effective theory. There is a singular point on the Coulomb branch at which the monopole becomes massless. In addition, once we introduce an equal mass for the electrons, there is another singularity on the moduli space at which the electrons are massless. When these singular points coincide with each other, the low energy effective theory contains a massless monopole and massless electrons with an Abelian gauge field [36,37]. This theory motivates us to consider a model with a monopole and electrons in Abelian gauge theory. Although we do not have an UV complete theory like their $N=2$ supersymmetric theory, we consider the model in a bottom-up approach to cosmological problems. (Although one might wonder whether the electric coupling constant blows up in the UV limit in our model, we expect that it asymptotically approaches an UV fixed point, as discussed in Ref. [36,37]).

Chiral symmetry breaking in $U(1)$ gauge theory.-We consider a nonsupersymmetric $U(1)_{H}$ gauge theory in this Letter. (However, its supersymmetric extension will be straightforward.) We introduce a scalar monopole $\phi$ and fermionic electrons, which are charged under a $U(1)_{H}$ gauge symmetry. We denote the unit electric and monopole charges as $e$ and $g$, respectively, which are related by the Dirac quantization condition

$$
e g=\frac{4 \pi}{2} n,
$$

where $n=0, \pm 1, \pm 2, \ldots$. We assume that $U(1)_{H}$ gauge symmetry is spontaneously broken by the condensation of the monopole,

$$
\langle\phi\rangle \equiv v,
$$

and the $U(1)_{H}$ gauge boson acquires an effective mass of $m_{v}=g v$. The spontaneous symmetry breaking of $U(1)_{H}$ symmetry implies the formation of cosmic strings. In fact, each electron and positron pair is connected with a cosmic string [33]. The tension of the string is calculated as

$$
\mu=\frac{e^{2}}{8 \pi} m_{v}^{2} \log \left(\frac{m_{m}^{2}}{m_{v}^{2}}+1\right),
$$

where $m_{m}$ is the mass of the monopole. Note that the tension is almost independent of $e$ and $g$ because of the Dirac quantization condition. Since each electron and positron pair is connected by the string, electrons and positrons are confined.

Here, let us consider a low energy effective theory below the confinement scale. Since we consider an Abelian gauge theory with fermionic electrons and positrons whose charges are unity, there are only boson states in the low energy. This implies that the theory cannot satisfy the 't Hooft anomaly matching condition to the chiral symmetry between the low and high energy scales unless the chiral symmetry is dynamically broken. Thus, we assume that the chiral symmetry is dynamically broken below the confinement scale.

Origin of $P Q$ symmetry.-Now we consider a theory with an extra dimension where 5D manifold is $R^{4} \times S / Z_{2}$. Two branes are located at the fixed points in the $S^{1} / Z_{2}$ orbifold, one of which the monopole as well as the standard model (SM) particles lives on. We introduce $N_{F}(\geq 4)$ pairs of electrons $Q^{i}$ and $\bar{Q}^{i}$ and put them separately on our brane and the other brane $[14,15]$. These electrons are charged under the hidden Abelian gauge symmetry $U(1)_{H}$ as shown in Table I. The $U(1)_{H}$ gauge field and the SM gauge fields propagate in the bulk. The first three $Q^{i}\left(\bar{Q}^{i}\right)$ of "flavor" indices $i=1,2,3$ transform under the fundamental (antifundamental) representation of $S U(3)_{c}$ as indicated as $3\left(\mathbf{3}^{*}\right)$ in Table I, so that we call them extra quarks (antiquarks). Since direct contact interactions between $Q$ and $\bar{Q}$ are suppressed exponentially due to the separation in the extra dimension $[14,15]$, there is an approximate chiral symmetry in our model.

As explained in the previous section, we consider that the chiral symmetry is dynamically broken at an intermediate scale $f_{a}(\approx v)$ :

$$
\left\langle Q^{i} \bar{Q}^{j}\right\rangle \simeq f_{a}^{3} \delta^{i j}
$$

If the QCD interaction were to be turned off, there would be $S U\left(N_{F}\right)_{L} \times S U\left(N_{F}\right)_{R} \times U(1)_{A}$ flavor symmetry before chiral symmetry breaking. [Note that the vector symmetry is just $U(1)_{H}$ gauge symmetry]. It is broken down to

TABLE I. Charge assignment for matter fields.

\begin{tabular}{lcccc}
\hline \hline & $Q^{i(=1,2,3)}$ & $\bar{Q}^{i(=1,2,3)}$ & $Q^{i(\geq 4)}$ & $\bar{Q}^{i(\geq 4)}$ \\
\hline$S U(3)_{c}$ & $\mathbf{3}$ & $\mathbf{3}^{*}$ & $\mathbf{1}$ & $\mathbf{1}$ \\
$U(1)_{H}$ & 1 & -1 & 1 & -1 \\
$U(1)_{\mathrm{PQ}}$ & 0 & 1 & 0 & $-\left(3 / N_{F}-3\right)$ \\
\hline \hline
\end{tabular}


$S U\left(N_{F}\right)_{V}$ by chiral symmetry breaking and $\left(N_{F}^{2}-1\right) \mathrm{NG}$ bosons may arise in the low energy effective theory. However, the flavor symmetry is explicitly broken by $S U(3)_{c}$ gauge interactions, so that $S U(3)_{c}$ charged NG bosons obtain masses via $S U(3)_{c}$ radiative corrections. The $U(1)_{A}\left[U(1)_{H}\right]^{2}$ anomaly induces an effective mass to the pseudo-NG boson associated with the $U(1)_{A}$ symmetry. (The pseudo-NG boson corresponding to the axial anomaly may obtain an effective mass by the Witten effect in the presence of the monopole [38,39]). Finally, the QCD axion can be identified with a pseudo-NG boson associated with the linear combination of axial symmetries shown in Table I as $U(1)_{\mathrm{PQ}}$ [20]. To sum up, there are $\left(N_{F}-3\right)^{2}-1$ massless NG bosons and an axion in the low energy effective theory. Note that when $N_{F}=4$, which is the minimal number of allowed values of $N_{F}$ in our model, there is only the axion in the low energy effective theory.

There are two advantages in this model. First, PQ symmetry is completely broken by a nonperturbative effect after the QCD phase transition, so that the domain wall number is unity. Since domain walls are unstable due to their tension in this case, there is no domain wall problem. Second, the $U(1)_{H}$ symmetry forbids operators containing either $Q$ or $\bar{Q}$, which arise and may be problematic in the original model with an $S U(N)_{H}$ gauge symmetry [14,15]. This also guarantees the precision of the PQ symmetry.

Here we comment on hadron states of electrons. There is no baryon state in the low energy because the confinement of electrons is induced by the Abelian $U(1)_{H}$ interaction [40]. There are heavy meson states in the low energy. In particular, composite states like $\pi^{i} \equiv\left(Q^{i} \bar{Q}^{4}\right)$ (hereafter, we take $i=1,2,3)$ may be stable heavy mesons, and their energy density may overclose the Universe. We can make them unstable in the following way $[15,45]$. First, we assume that the fields $Q^{i}$ and $\bar{Q}^{i}$ have the same SM gauge charge with the right-handed down quark and its complex conjugate, respectively, which is also motivated by grand unified theories. We also introduce a heavy scalar $\chi^{i}$ which lives on our brane (like $Q^{i}$ ) and has the same SM gauge charge with $\bar{Q}^{i}$ [46]. Now we can write dimension 5 interactions of $\left[\left(Q^{4}\right)^{\dagger} \gamma^{\mu} Q^{i}\right] D_{\mu} \chi_{i}+$ c.c., where $D_{\mu}$ is the covariant derivative. After chiral symmetry breaking these interactions become the operators of $D_{\mu} \pi^{i} D_{\mu} \chi_{i}+$ c.c., which implies kinetic mixing between $\pi^{i}$ and $\chi^{i}$. Once we make the field $\chi^{i}$ decay into SM particles via Yukawa interactions of $\epsilon_{i j k} \chi^{i} q_{L}^{j} q_{L}^{k}+$ c.c., where $q_{L}$ is the lefthanded quark, the heavy meson $\pi^{i}$ also decays via kinetic mixing [47]. The above interactions never violate the PQ symmetry defined in Table I because we can introduce the dimension 5 interactions only on our brane, where the SM particles, $Q^{i}$, and $\chi^{i}$ live.

Predictions.-Since the PQ symmetry is dynamically broken after inflation, a cosmic string and domain wall system arises after the QCD phase transition. In our model, the domain wall number is unity, so that the system is short lived and disappears soon after it forms. Axions are generated from the decay of these topological defects as well as from the usual misalignment mechanism [48-50]. Here, we should take the average in the initial angle of axion because it randomly distributes in the phase space. To sum up, the axion abundance is given by [51]

$$
\Omega_{a} h^{2} \simeq(1.7 \pm 0.4) \times 10^{-2} \times\left(\frac{f_{a}}{10^{10} \mathrm{GeV}}\right)^{1.19} .
$$

The observed DM abundance implies that the axion decay constant is given by

$$
f_{a} \simeq(4.2-6.5) \times 10^{10} \mathrm{GeV} .
$$

Note that this decay constant corresponds to the axion mass of

$$
m_{a} \simeq(0.9-1.4) \times 10^{-4} \mathrm{eV} .
$$

Notably, the Axion Dark Matter Experiment (ADMX) will be improved by the use of higher harmonic ports and will cover the axion mass range of $16-160 \mu \mathrm{eV}$ [52].

There are $\left(N_{F}-3\right)^{2}-1 \mathrm{NG}$ bosons as well as an axion in the low energy effective theory. Since PQ symmetry is broken after inflation, the bosons and the axion are in thermal equilibrium and then decouple from the thermal plasma after PQ symmetry breaking $[53,54]$. Their abundance is determined by the conservation of entropy density and is conventionally expressed by the effective neutrino number as [55-57]

$$
N_{\text {eff }} \simeq N_{\text {eff }}^{(\mathrm{SM})}+0.027 \times\left(N_{F}-3\right)^{2},
$$

where $N_{\mathrm{eff}}^{(\mathrm{SM})}(\simeq 3.046)$ is the SM prediction. The present constraint is $N_{\text {eff }}=2.99 \pm 0.39$ (95\% C.L.) [58], so that $N_{F}$ has to be smaller than or equal to 6 . The ground-based stage-IV CMB polarization experiment CMB-S4 will measure the effective neutrino number with a precision of $\Delta N_{\text {eff }}=0.0156$ within one sigma level $[59,60]$. If the number of flavors would be measured via the observation of the effective neutrino number, it would be remarkable evidence for our model. Unfortunately, however, our model cannot distinguish with the Kim-Shifman-VainshteinZakharov axion model in the case of $N_{f}=4$ [61].

Discussion and conclusions. - We have proposed a QCD axion model where PQ symmetry accidentally arises due to the separation of extra quark and antiquark pairs in an extra dimension. In contrast to the original theory proposed in Refs. [14,15], we avoid the axion domain wall problem by assuming chiral symmetry breaking in an Abelian gauge theory. In fact, there are some proposals that Abelian gauge theories develop confinement and chiral symmetry breaking, including 't Hooft's conjecture of Abelian dominance [27], which is supported by lattice QCD [28-30], and the confinement theory by monopole condensation in an 
Abelian theory [33]. There are also UV complete models with electrons and monopoles in an Abelian gauge theory in $N=2$ supersymmetry [34-37]. Motivated by these studies, we consider an Abelian gauge theory with chiral symmetry breaking of electrons triggered by a monopole condensation. Since we consider an Abelian theory rather than non-Abelian theories, PQ symmetry is completely broken at the QCD phase transition and domain walls disappear soon after they form at that time.

Finally, we comment on another possibility to realize chiral symmetry breaking in an Abelian gauge theory. Suppose that there are monopoles but no electron in an Abelian gauge theory. This is equivalent to the QED via dual symmetry, so that the monopole charge is asymptotically nonfree. Then, let us add electrons with an electric charge satisfying Dirac's quantization condition. When the number of electrons is much smaller than that of the monopoles, their effect on the beta function is negligible. This implies that the monopole charge remains asymptotically nonfree at least until the monopole charge becomes much smaller than the electron charge. Then Dirac's quantization condition implies that the electric charge increases as the energy scale decreases; i.e., it is asymptotic free. As the electric charge increases, its effect on the beta function becomes efficient and the beta function may be canceled and suppressed. Then there may be a certain value of electric charge at which the beta function is absent. In Ref. [62], the self-energy of chiral fermion has been calculated and it has been shown that chiral symmetry breaking occurs in an Abelian gauge theory when the gauge coupling is larger than the threshold value of $e_{c}=2 \pi / \sqrt{3}$. This implies that Abelian gauge theory can realize the chiral condensation when the number of electrons is sufficiently smaller than that of the monopoles.

T. T. Y thanks H. Murayama and Y. Tachikawa for the discussions and comments. M. Y. thanks W. Buchmüller for the kind hospitality at DESY, where this work was finished. This work is supported by a Grant-in-Aid for Scientific Research from the Ministry of Education, Science, Sports, and Culture (MEXT), Japan, Grants No. 26104009 and No. 26287039 (T. T. Y), the World Premier International Research Center Initiative (WPI Initiative), MEXT, Japan, and the Program for the Leading Graduate Schools, MEXT, Japan (M. Y.). M. Y. acknowledges the support from the JSPS Research Fellowships for Young Scientists, Grant No. 25.8715 .

[1] C. A. Baker, D. D. Doyle, P. Geltenbort, K. Green, M. G. D. van der Grinten, P. G. Harris, P. Iaydjiev, S. N. Ivanov et al., Improved Experimental Limit on the Electric Dipole Moment of the Neutron, Phys. Rev. Lett. 97, 131801 (2006).

[2] R. D. Peccei and H. R. Quinn, $C P$ Conservation in the Presence of Instantons, Phys. Rev. Lett. 38, 1440 (1977).
[3] R. D. Peccei and H. R. Quinn, Constraints imposed by $C P$ conservation in the presence of instantons, Phys. Rev. D 16, 1791 (1977).

[4] S. Weinberg, A New Light Boson?, Phys. Rev. Lett. 40, 223 (1978); F. Wilczek, Problem of Strong $p$ and $t$ Invariance in the Presence of Instantons, Phys. Rev. Lett. 40, 279 (1978).

[5] G. 't Hooft, Symmetry Breaking through Bell-Jackiw Anomalies, Phys. Rev. Lett. 37, 8 (1976).

[6] G. 't Hooft, Computation of the quantum effects due to a four-dimensional pseudoparticle, Phys. Rev. D 14, 3432 (1976); 18, 2199(E) (1978).

[7] S. B. Giddings and A. Strominger, Loss of incoherence and determination of coupling constants in quantum gravity, Nucl. Phys. B307, 854 (1988).

[8] S. R. Coleman, Why there is nothing rather than something: A theory of the cosmological constant, Nucl. Phys. B310, 643 (1988).

[9] G. Gilbert, Wormhole induced proton decay, Nucl. Phys. B328, 159 (1989).

[10] T. Banks and N. Seiberg, Symmetries and strings in field theory and gravity, Phys. Rev. D 83, 084019 (2011).

[11] M. Kamionkowski and J. March-Russell, Planck scale physics and the Peccei-Quinn mechanism, Phys. Lett. B 282, 137 (1992).

[12] S. Ghigna, M. Lusignoli, and M. Roncadelli, Instability of the invisible axion, Phys. Lett. B 283, 278 (1992).

[13] B. A. Dobrescu, Strong $C P$ problem versus Planck scale physics, Phys. Rev. D 55, 5826 (1997).

[14] K. I. Izawa, T. Watari, and T. Yanagida, Higher dimensional QCD without the strong $C P$ problem, Phys. Lett. B 534, 93 (2002).

[15] K. I. Izawa, T. Watari, and T. Yanagida, Supersymmetric and $C P$-symmetric QCD in higher dimensions, Phys. Lett. B 589, 141 (2004).

[16] R. Holman, S. D. H. Hsu, T. W. Kephart, E. W. Kolb, R. Watkins, and L. M. Widrow, Solutions to the strong $C P$ problem in a world with gravity, Phys. Lett. B 282, 132 (1992).

[17] H. C. Cheng and D. E. Kaplan, Axions and a gauged PecceiQuinn symmetry, arXiv:hep-ph/0103346.

[18] S. M. Barr and D. Seckel, Cosmological constant, false vacua, and axions, Phys. Rev. D 64, 123513 (2001).

[19] K. Harigaya, M. Ibe, K. Schmitz, and T. T. Yanagida, Peccei-Quinn symmetry from dynamical supersymmetry breaking, Phys. Rev. D 92, 075003 (2015).

[20] J. E. Kim, Composite invisible axion, Phys. Rev. D 31, 1733 (1985).

[21] M. Axenides, R. H. Brandenberger, and M. S. Turner, Development of axion perturbations in an axion dominated universe, Phys. Lett. 126B, 178 (1983).

[22] D. Seckel and M. S. Turner, Isothermal, density perturbations in an axion-dominated inflationary universe, Phys. Rev. D 32, 3178 (1985).

[23] M. S. Turner and F. Wilczek, Inflationary Axion Cosmology, Phys. Rev. Lett. 66, 5 (1991).

[24] M. Kawasaki, M. Yamada, and T. T. Yanagida, Cosmologically safe QCD axion as a present from extra dimension, Phys. Lett. B 750, 12 (2015).

[25] Y. B. Zeldovich, I. Y. Kobzarev, and L. B. Okun, Cosmological consequences of the spontaneous breakdown of 
discrete symmetry, Zh. Eksp. Teor. Fiz. 67, 3 (1974) [Sov. Phys. JETP 40, 1 (1974)].

[26] P. Sikivie, Axions, Domain Walls, and the Early Universe, Phys. Rev. Lett. 48, 1156 (1982).

[27] G. 't Hooft, Topology of the gauge condition and new confinement phases in non-Abelian gauge theories, Nucl. Phys. B190, 455 (1981).

[28] J. D. Stack, S. D. Neiman, and R. J. Wensley, String tension from monopoles in SU(2) lattice gauge theory, Phys. Rev. D 50, 3399 (1994).

[29] O. Miyamura, Chiral symmetry breaking in gauge fields dominated by monopoles on SU(2) lattices, Phys. Lett. B 353, 91 (1995).

[30] R. M. Woloshyn, Chiral symmetry breaking in Abelian projected SU(2) lattice gauge theory, Phys. Rev. D 51, 6411 (1995).

[31] A. S. Kronfeld, G. Schierholz, and U. J. Wiese, Topology and dynamics of the confinement mechanism, Nucl. Phys. B293, 461 (1987).

[32] A. S. Kronfeld, M. L. Laursen, G. Schierholz, and U. J. Wiese, Monopole condensation and color confinement, Phys. Lett. B 198, 516 (1987).

[33] Y. Nambu, Strings, monopoles, and gauge fields, Phys. Rev. D 10, 4262 (1974); G. 't Hooft, in High Energy Physics edited by A. Zichichi (Editorice Compositori, Bologna, 1975); S. Mandelstam, Vortices and quark confinement in non-Abelian gauge theories, Phys. Rep. 23, 245 (1976).

[34] N. Seiberg and E. Witten, Electric-magnetic duality, monopole condensation, and confinement in $N=2$ supersymmetric Yang-Mills theory, Nucl. Phys. B426, 19 (1994); B430, 485(E) (1994).

[35] N. Seiberg and E. Witten, Monopoles, duality and chiral symmetry breaking in $N=2$ supersymmetric QCD, Nucl. Phys. B431, 484 (1994).

[36] P. C. Argyres and M. R. Douglas, New phenomena in SU(3) supersymmetric gauge theory, Nucl. Phys. B448, 93 (1995).

[37] P. C. Argyres, M. R. Plesser, N. Seiberg, and E. Witten, New $N=2$ superconformal field theories in four dimensions, Nucl. Phys. B461, 71 (1996).

[38] E. Witten, Dyons of charge $e \theta / 2 \pi$, Phys. Lett. 86B, 283 (1979).

[39] W. Fischler and J. Preskill, Dyon-axion dynamics, Phys. Lett. 125B, 165 (1983).

[40] One might wonder whether there are stable topological solitons called skyrmions in the low energy effective theory. We assume that they are unstable due to some dynamical operator that breaks topological charge conservation similar to monopole operators in three dimensions [41,42] or instanton operators in five dimensions [43]. Breaking of a $U(1)$ symmetry in the IR theory which is absent in the UV theory by such an operator may be quite common in strong dynamics (see, e.g., Ref. [44]).

[41] G. 't Hooft, On the phase transition towards permanent quark confinement, Nucl. Phys. B138, 1 (1978).

[42] V. Borokhov, A. Kapustin, and X.k. Wu, Topological disorder operators in three-dimensional conformal field theory, J. High Energy Phys. 11 (2002) 049.

[43] N. Lambert, C. Papageorgakis, and M. Schmidt-Sommerfeld, Instanton operators in five-dimensional gauge theories, J. High Energy Phys. 03 (2015) 019.
[44] O. Aharony, S. S. Razamat, N. Seiberg, and B. Willett, 3D dualities from 4D dualities, J. High Energy Phys. 07 (2013) 149.

[45] Instead, one can assume that these heavy mesons are washed out by the primordial inflation and are never produced by a reheating process. This is the case if the reheating temperature is much lower than the dynamical scale.

[46] In the supersymmetric extension, we can identify the field $\chi^{i}$ with a scalar down-type quark, as pointed out in Ref. [15]. The scalar quark can decay quickly if the SM gauginos are lighter than the scalar quark.

[47] One might wonder if the Yukawa interactions lead to dangerous proton decay. However, if the mass of $\chi^{i}$ is of the order $10^{10} \mathrm{GeV}$ and the Yukawa coupling constants to the first family quarks and leptons are $O\left(10^{-6}\right)$, the proton lifetime is consistent with the present lower bound. If $\chi^{i}$ couples to the third family quarks and leptons with Yukawa couplings of $O(1)$, the decay temperature of $\pi^{i}$ is as large as $10^{5} \mathrm{GeV}$ and its energy density never dominates the Universe.

[48] J. Preskill, M. B. Wise, and F. Wilczek, Cosmology of the invisible axion, Phys. Lett. 120B, 127 (1983).

[49] L. F. Abbott and P. Sikivie, A cosmological bound on the invisible axion, Phys. Lett. 120B, 133 (1983).

[50] M. Dine and W. Fischler, The not so harmless axion, Phys. Lett. 120B, 137 (1983).

[51] M. Kawasaki, K. Saikawa, and T. Sekiguchi, Axion dark matter from topological defects, Phys. Rev. D 91, 065014 (2015).

[52] K. van Bibber and G. Carosi, Status of the ADMX and ADMX-HF experiments, arXiv:1304.7803.

[53] M. S. Turner, Thermal Production of Not So Invisible Axions in the Early Universe, Phys. Rev. Lett. 59, 2489 (1987); 60, 1101(E) (1988).

[54] A. Salvio, A. Strumia, and W. Xue, Thermal axion production, J. Cosmol. Astropart. Phys. 01 (2014) 011.

[55] K. Nakayama, F. Takahashi, and T. T. Yanagida, A theory of extra radiation in the Universe, Phys. Lett. B 697, 275 (2011).

[56] S. Weinberg, Goldstone Bosons as Fractional Cosmic Neutrinos, Phys. Rev. Lett. 110, 241301 (2013).

[57] M. Kawasaki, M. Yamada, and T. T. Yanagida, Observable dark radiation from cosmologically safe QCD axion, Phys. Rev. D 91, 125018 (2015).

[58] P. A. R. Ade et al. (Planck Collaboration), Planck 2015 results. XIII. Cosmological parameters, arXiv:1502.01589.

[59] K. N. Abazajian et al., Neutrino physics from the cosmic microwave background and large scale structure, Astropart. Phys. 63, 66 (2015).

[60] W. L. K. Wu, J. Errard, C. Dvorkin, C. L. Kuo, A. T. Lee, P. McDonald, A. Slosar, and O. Zahn, A guide to designing future ground-based cosmic microwave background experiments, Astrophys. J. 788, 138 (2014).

[61] J. E. Kim, Weak Interaction Singlet and Strong CP Invariance, Phys. Rev. Lett. 43, 103 (1979); M. A. Shifman, A. I. Vainshtein, and V. I. Zakharov, Can confinement ensure natural $C P$ invariance of strong interactions?, Nucl. Phys. B166, 493 (1980).

[62] T. Akiba and T. Yanagida, Hierarchic chiral condensate, Phys. Lett. 169B, 432 (1986). 